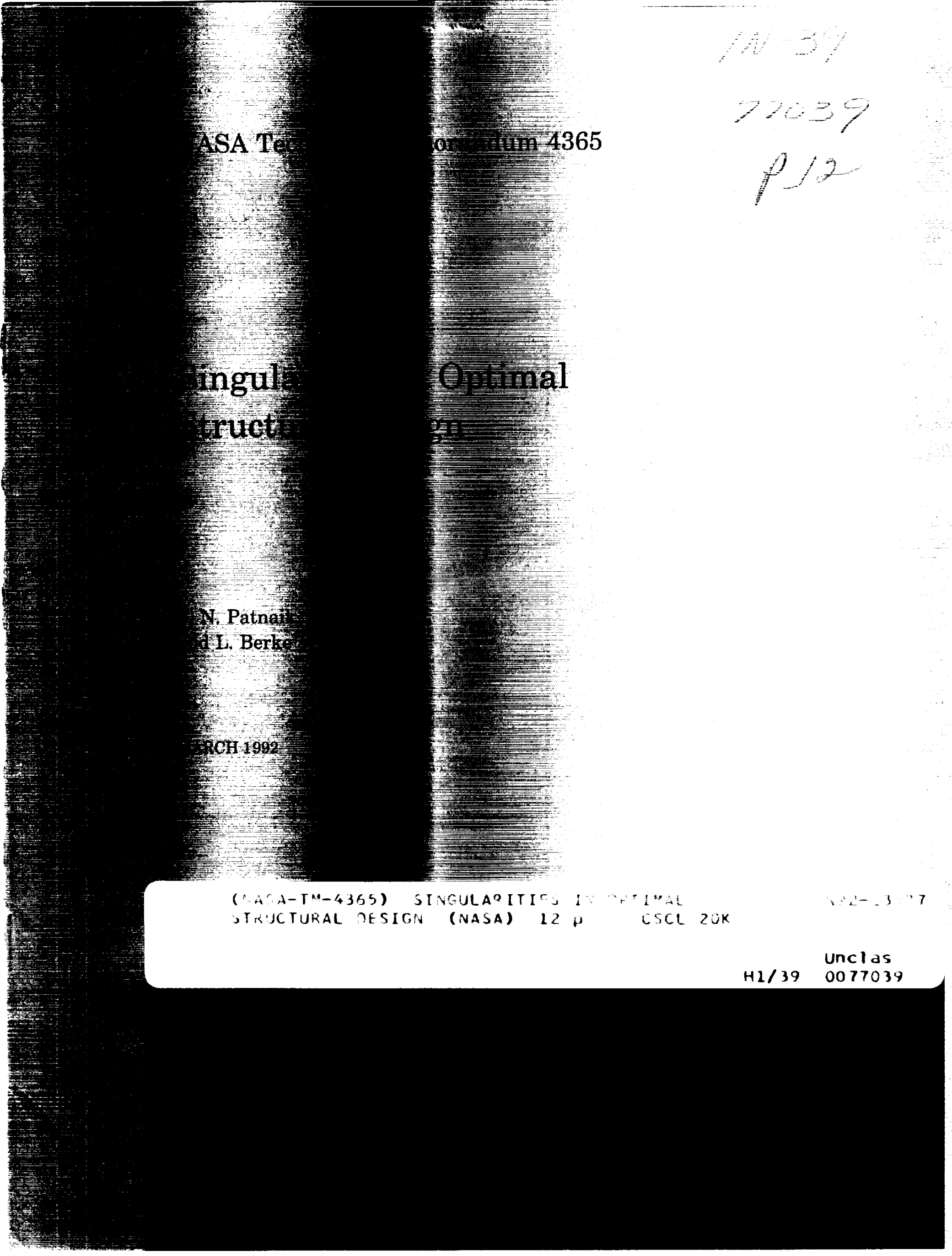




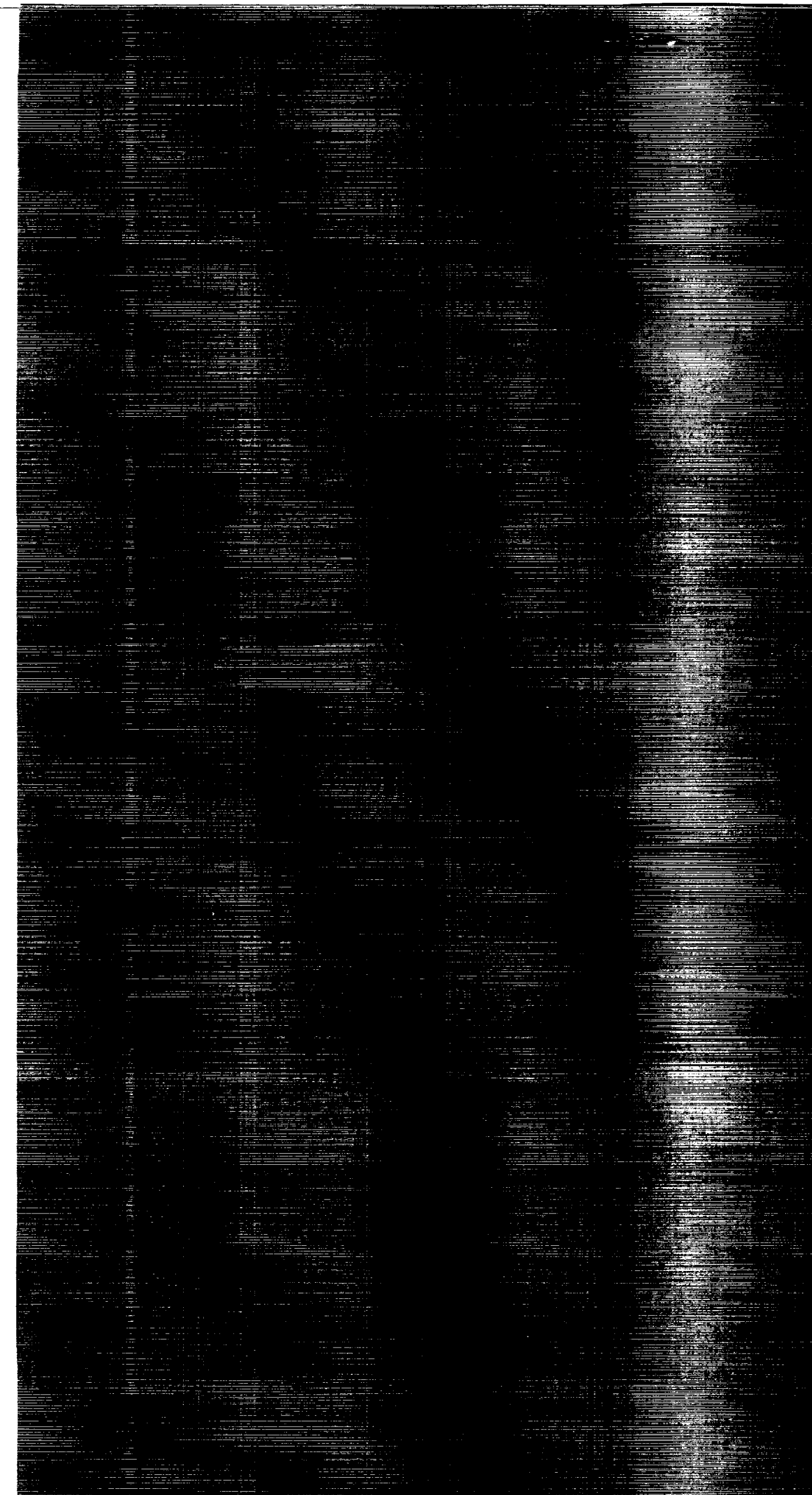


NASA Technical Memorandum 4365

\section{Singularities in Optimal Structural Design}

S. N. Patnaik

Ohio Aerospace Institute

Brook Park, Ohio

J. D. Guptill and L. Berke

Lewis Research Center

Cleveland, Ohio

\section{N/SA}

National Aeronautics and

Space Administration

Office of Management

Scientific and Technical

Information Program

1992 


\section{Summary}

Singularity conditions that arise during structural optimization can seriously degrade the performance of the optimizer. The singularities are intrinsic to the formulation of the structural optimization problem and are not associated with the method of analysis. Certain conditions that give rise to singularities have been identified in earlier papers, along with a proposition to alleviate the consequences of their presence (refs. 1 to 3 ). These singularities were global in nature, encompassing the entire structure. Further examination revealed more complex sets of conditions in which singularities occur. Some of these singularities are local in nature, being associated with only a segment of the structure. Moreover, the likelihood that one of these local singularities may arise during an optimization procedure can be much greater than that of the global singularity identified earlier. This paper provides examples of these additional forms of singularities. It also gives a framework in which these singularities can be recognized. In particular, the singularities can be identified by examination of the stressdisplacement relations along with the compatibility conditions and/or the displacement-stress relations derived in the integrated force method of structural analysis.

\section{Introduction}

Structural optimization methodologies based on mathematical programming techniques require constraint-gradient information (ref. 4). Nonlinear optimization proceeds iteratively, and at each iteration a direction vector $\vec{\phi}$ is generated in the design variable space. The formation of this direction vector utilizes the gradients. Consider, for example, the coefficient matrix $[\mathrm{H}]$ used in a method of feasible directions, as given by Best, (ref. 4) to find the direction vector

$$
\phi=\frac{[H] \vec{\nabla} f}{2 \lambda_{0}}
$$

where

$$
\left.|H|=[I]-\left[\nabla g||[\nabla g]^{T} \mid \nabla g\right]\right]^{-1}[\nabla g]^{T}
$$

$$
|\nabla g|=\left[\begin{array}{cccc}
\frac{\partial g_{1}}{\partial \chi_{1}} & \frac{\partial g_{2}}{\partial \chi_{1}} & \ldots & \frac{\partial g_{k}}{\partial \chi_{1}} \\
\frac{\partial g_{1}}{\partial \chi_{2}} & \frac{\partial g_{2}}{\partial \chi_{2}} & \ldots . & \frac{\partial g_{k}}{\partial \chi_{2}} \\
& \vdots & \\
\frac{\partial g_{1}}{\partial \chi_{n}} & \frac{\partial g_{2}}{\partial \chi_{n}} & \ldots & \frac{\partial g_{k}}{\partial \chi_{n}}
\end{array}\right]
$$

where $\vec{\nabla} f$ is the gradient of the merit function,

$$
\lambda_{0}=-0.5 \sqrt{|[H] \vec{\nabla} f|^{T}[H] \vec{\nabla} f}
$$

and $\kappa$ is the number of active constraints used in the computation of the direction vector.

In equation (3), $\chi_{i}$ represents the $i$ th design variable, and $g_{j}$ represents the $j$ th constraint value (e.g.. the nondimensionalized element stress or nodal displacement). Here, we consider only sizing design variables (e.g., the cross-sectional areas of truss elements).

Now, if the rank of the gradient matrix $[\nabla g]$ is less than $\kappa$, then the derived composite matrix $\left.[\mid \nabla g]^{T_{T}} \mid \nabla g\right] \mid$ will be singular. Thus, the coefficient matrix $[H]$ is not defined, and any direction generated from equation (1) would be spurious. Gradient projection methods that use the matrix $[H]$ can suffer the same consequences, and similar remarks can be made about other techniques that use constraint-gradient information in this way. Computer codes can react to this situation by premature termination (sometimes with indications of multiple overflow errors) or divergence. These observations are intrinsic to the specification of the constraint formulation in the structural optimization problem and are not associated with any specific analyzer.

Clearly, the rank of the gradient matrix $[\nabla g]$ is less than or equal to the smaller of the number of active constraints $k$ and the number of design variables $n$. Thus, whenever the 
number of active constraints is larger than the number of design variables, the rank of $[\nabla g]$ will be less than $\kappa$ (since $n<\kappa)$, and singularities in $[H]$ will occur. However, as pointed out earlier (refs. I to 3), whenever the number of active stress and displacement constraints is more than the number of displacement degrees of freedom of the structure (which is often significantly less than $n$ ), singularities will also be introduced. This condition depends only on the number of active constraints, irrespective of where on the structure the active constraints appear, and will be referred to as a global singularity condition.

\section{Linear Functional Dependence Among Constraints}

Constraint sets formed during structural optimization may exhibit functional dependence. Moreover, this functional dependence may be linear in form. That is, for certain sets of constraint functions of the design variable $\vec{\chi}\left(\right.$ e.g., $\left\{g_{1}(\vec{\chi})\right.$, $\left.\left.g_{2}(\vec{\chi}), \ldots, g_{k}(\vec{x})\right\}\right)$, there exists a set of constants $\left\{\alpha_{j}\right\}$, not all zero, such that

$$
\sum_{j=1}^{\kappa} \alpha_{j} g_{j}(\vec{\chi})=0 \quad \text { for all } \vec{\chi}
$$

Note that any individual function $g_{j}(\vec{x})$ may be nonlinear in the independent variable $\vec{\chi}$. This linear functional dependence, in turn, generates linear dependence among constraint gradients (which leads to singularities). Differentiating equation (5) with respect to $\vec{\chi}$ gives

$$
\sum_{j=1}^{\kappa} \alpha_{j} \nabla g_{j}=0
$$

where $\nabla g_{j}$ is the gradient of the function $g_{j}$ with respect to $\vec{\chi}$.

\section{Constraint Dependence for a Three-Bar Truss}

To illustrate the existence and importance of linear functional dependence among constraints, consider a three-bar steel truss with a Young's modulus $E$ of $30000 \mathrm{ksi}$ and a strength $\sigma_{0}$ of $20 \mathrm{ksi}$ (fig. 1). For each load condition, the truss can have three stress and two displacement constraints. The stresses and displacements are functions of the cross-sectional areas of the bar elements $\bar{\chi}$ and either (or both) type(s) of constraints may appear in the active constraint set. It can be shown that, for the three-bar truss, the following relationships hold for each load condition:

$$
\begin{gathered}
X_{1}=\left(\frac{1}{E}\right)\left(\ell \sigma_{2}-2 \ell \sigma_{1}\right) \\
X_{2}=\left(\frac{1}{E}\right)\left(\ell \sigma_{2}\right) \\
\left(\sigma_{1}-\sigma_{2}+\sigma_{3}\right)=0.0
\end{gathered}
$$

where $X_{j}(\vec{\chi})$, the $j$ th nodal displacement, and $\sigma_{i}(\vec{\chi})$, the $i$ th element stress, are related to the behavior constraints and are functions of the three-component design variable $\vec{\chi}$ (the areas of the elements). Here, $l$ is the length of the second element (see fig. 1).

Note that these relationships hold for all values of the design variable (since $\chi_{i}$ does not appear explicitly in eqs. (7)). Furthermore, linear functional dependence (or independence) of a set of behavior variables $\left\{\sigma_{i}, X_{j}\right\}$ implies the linear dependence (or independence) of their associated constraint gradients (ref. 5).

Since we have three equations in five unknown functions $\left(\sigma_{1}, \sigma_{2}, \sigma_{3}, X_{1}\right.$, and $\left.X_{2}\right)$, given any two of the five functions (except for the $\sigma_{2}, X_{2}$ pair), the other three functions can be determined. The observation that any set of more than two constraints is linearly functionally dependent corresponds to
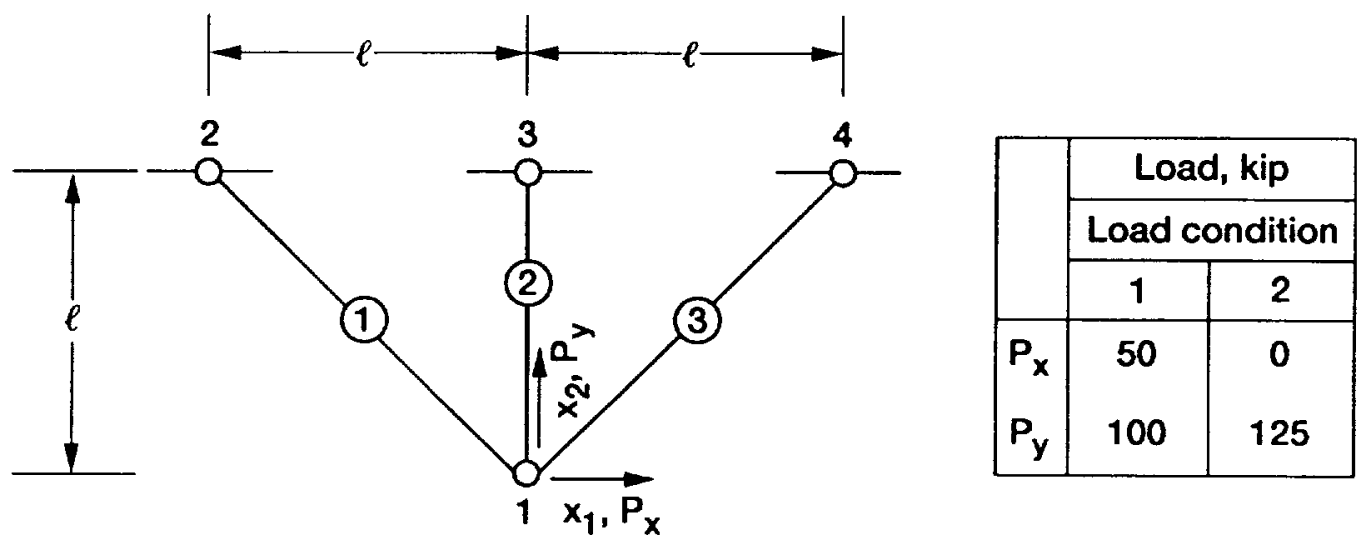

Figure 1.-Three-bar truss. (Elements are circled, nodes are not.) 
TABLE I.--RANKS OF SEVERAL CONSTRAINT-GRADIENT MATRICES FOR THE THREE-BAR TRUSS

|Element areas: $1.00,9.30$, and 3.67 in. $^{2}$ ]

\begin{tabular}{|c|c|c|c|c|}
\hline \multirow[t]{2}{*}{ Case } & \multicolumn{2}{|c|}{ Constraint set } & \multirow{2}{*}{$\begin{array}{l}\text { Rank of } \\
\text { constraint- } \\
\text { gradient } \\
\text { matrix }\end{array}$} & \multirow{2}{*}{$\begin{array}{l}\text { Nature of } \\
\text { singularity }\end{array}$} \\
\hline & Number & Constraints & & \\
\hline $\begin{array}{l}1 \\
2 \\
3\end{array}$ & $\begin{array}{l}5 \\
3 \\
2\end{array}$ & $\begin{array}{c}\left\{\sigma_{1}, \sigma_{2}, \sigma_{3}, X_{1}, X_{2}\right\} \\
\left\{\sigma_{1}, \sigma_{2}, \sigma_{3}\right\} \\
\left.\mid \sigma_{2}, X_{2}\right\}\end{array}$ & $\begin{array}{l}2 \\
2 \\
1\end{array}$ & $\begin{array}{c}\text { Global } \\
\text { Global and local } \\
\text { Local }\end{array}$ \\
\hline
\end{tabular}

the global singularity condition identified earlier (refs. 1 to 3 ). The linear functional dependence among stresses, also noted earlier (refs. 2 and 3), is clearly indicated by equation (7b). Use of the knowledge of this global singularity to restrict the active constraint set to two constraints is, however, insufficient to guarantee linear functional independence. In particular, equation (7a.2) involves only one element stress $\left(\sigma_{2}\right)$ and one nodal displacement $\left(X_{2}\right)$ that would produce a singularity because of their linear functional dependence. We would expect, then, that the rank of the constraint-gradient matrix formed by (1) taking all five constraints would be two, (2) taking the three stress constraints would certainly be two, and (3) taking $\sigma_{2}$ and $X_{2}$ would be one. Table I shows the results of performing a singular value decomposition on the three constraint-gradient matrices indicated.

\section{Use of the IFM Equations for Identification of Singularities}

The integrated force method (IFM) is a structural analysis tool based on the method of forces through which global singularities in structural optimization were identified earlier (refs. 1 to 3). It will be used here to identify additional types of singularitics.

Let us consider, more generally, an arbitrary truss under a single load condition that has $n$ stress degrees of freedom and $m$ displacement degrees of freedom. We will designate this structure as truss $(n, m)$, following the convention in the IFM (ref. 6). The following relations can be derived from the governing equations used in the IFM (see appendix B):

$$
\begin{aligned}
& \vec{\sigma}=[\tilde{B} \mid \vec{X} \\
& \vec{X}=[\tilde{J}] \vec{\sigma} \\
& \overrightarrow{0}=[\tilde{C}] \vec{\sigma}
\end{aligned}
$$

where the matrices $[\tilde{B}],[\tilde{J}]$, and $[\tilde{C}]$ are independent of the design variables; have full ranks of $m, m$, and $r$, respectively; have dimensions of $n \times m, m \times n$, and $r \times n$, respectively; and $r=n-m$. The choice of design variables depends on the structural elements chosen for the design, but typically these variables are taken as the element cross-sectional areas for truss elements, the moments of inertia for flexural elements, and the shear area and polar moment of inertia for elements with torsion.

Equation (8a) is referred to as the set of stress-displacement relations and is derived from the force equilibrium equations. Equation (8b) describes the displacement-stress relations. Equation ( $8 c)$ is the set of stress compatibility conditions. We note here that equations $(8 b)$ and $(8 c)$ can be used to derive equation ( $8 \mathrm{a})$, and vice-versa. From the stress compatibility conditions (eq. (8c)), we can see that the set of all stress constraints is linearly functionally dependent. These equations are also consistent with the observation regarding global singularities made earlier (that the maximum number of stress and displacement constraints which can form a set of linearly independent functions is $m$ ) (ref. 1). However, more subtle cases of singularities can arise by linear functional dependence among subsets of stress constraints (see eq. (8c)) or stress and displacement constraints (see eq. (8a) or (8b)). These singularities will be referred to as local singularities, since the relations are derived by consideration of local segments of the structure. This localization is reflected in the banded nature of the equilibrium and compatibility matrices, $|\tilde{B}|$ and $[\tilde{C}]$. respectively.

\section{Constraint Dependence for a 20-Bay Truss}

For a more comprehensive example of how easily these more subtle forms of singularities can arise, consider the 20 -bay truss shown in figure 2 . The truss $(101,80)$ consists of 20 bays, each defined by six adjunct elements. The truss has 101 elements and has 101 stress and 80 displacement degrees of freedom. It has 21 compatibility conditions $(n-m=21)$. Using the evidence found earlier of possible global singularities, one should restrict the set of active constraints to be no more than the number of displacement degrees of freedom $(m=80)$. The first two cases in table II show the rank of the resulting gradient matrix when more than 80 constraints are active. One might consider the restriction of limiting the number of active constraints to 80 (out of the maximum of 181 prescribed behavior constraints) to be rather mild since it might be expected that fewer than 80 constraints would be active, anyway. However, this restriction is insufficient to prevent all singularities that can arise

Consider, first, the stress-displacement relations of the structure, which are defined through the modified equilibrium matrix $[\tilde{B}]$. A few typical stress-displacement relations for the truss $(101,80)$ are as follows:

$$
\begin{gathered}
\sigma_{1}=\tilde{b}_{1.2} X_{2} \\
\sigma_{4}=\tilde{b}_{4.5} X_{5}+\tilde{b}_{4.6} X_{6} \\
\sigma_{88}=\tilde{b}_{88.69} X_{69}+\tilde{b}_{88.70} X_{70}+\tilde{b}_{88.71} X_{71}+\tilde{b}_{88.72} X_{72} \\
\sigma_{90}=\tilde{b}_{90.69} X_{69}+\tilde{b}_{90.73} X_{73}
\end{gathered}
$$

From Figure 2, we can see that the stress in element $1, \sigma_{1}$, and the vertical displacement at node $2, X_{2}$, are directly 


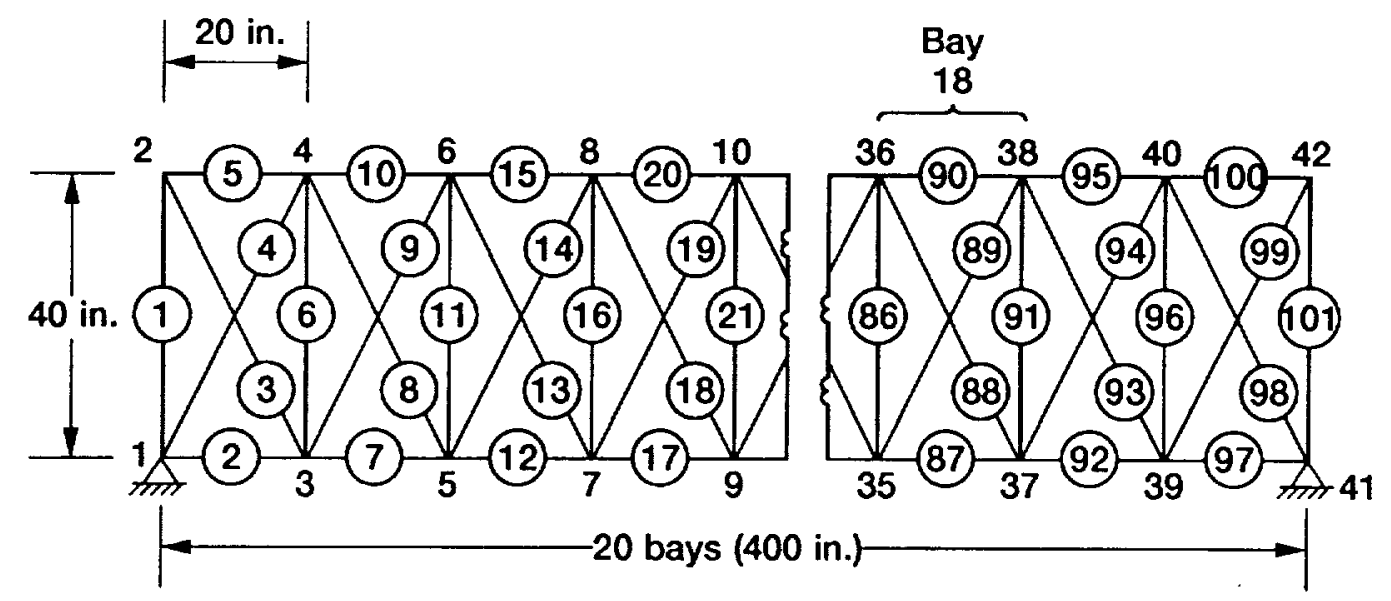

(a)

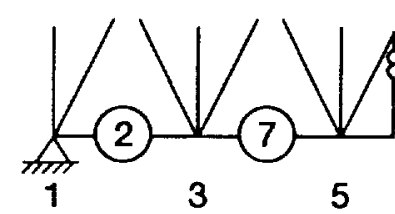

(b)

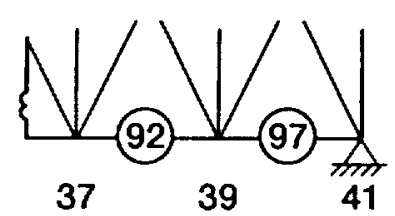

(a) Overall view

he flexural load path under gravity load.

(b) View showing the flexural load path
(c) View of bay 2.

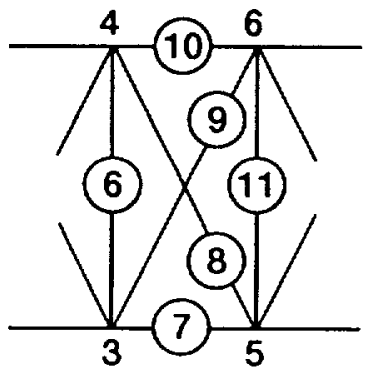

(c)

Figure 2. -20-bay truss. Displacement constraint numbering for all nodes $t$ such that $2 \leq 1 \leq 40$ is as follows: (1) for horizontal displacements, the constraint index is $2(1-1)-1 ;(2)$ for vertical displacements, the constraint index is $2(t-1)$. (Elements are circled, nodes are not.)

related as in equation (9a). Similarly, equation $(9 \mathrm{c})$ can be derived by examining the connectivity of element 88 in the 18 th bay in figure 2 . The stress in that element $\sigma_{88}$ can be determined from the horizontal and vertical components of the displacements at node $36, X_{69}$ and $X_{70}$, and at node $37, X_{71}$ and $X_{72}$. The other relations can be derived in a similar way. Since each relation is derived by examining a small subset of the structure, the bandwidth of the $[\tilde{B}]$ matrix will be small, and the singularities produced can be considered to be local singularities.

The element areas, which are the design variables for this problem, do not appear explicitly in equations (9), since the coefficients are independent of those areas. Taking, as examples, the sets of constraints $\left\{\sigma_{1}, X_{2}\right\},\left\{\sigma_{4}, X_{5}, X_{6}\right\},\left\{\sigma_{88}, X_{69}\right.$, $\left.X_{70}, X_{71}, X_{72}\right\}$, and $\left\{\sigma_{90}, X_{69}, X_{73}\right\}$ as active constraint sets, would produce rank-deficient gradient matrices $[\nabla g]$ and singular $[H]$ matrices (see cases 3 to 6 in table II for numerical verification). These clearly demonstrate that subsets containing many fewer active constraints than the global singularity restriction of at most 80 stress and displacement constraints can cause singularities. Next, consider the stress compatibility conditions (CC) of the truss $(101,80)$, which includes one external $\mathrm{CC}$ and 20 individual bay $\mathrm{CC}$. The external $\mathrm{CC}$, which has 20 terms, has the following form:

$$
\tilde{c}_{1,2} \sigma_{2}+\tilde{c}_{1,7} \sigma_{7}+\tilde{c}_{1,12} \sigma_{12}+\ldots,+\tilde{c}_{1,97} \sigma_{97}=0
$$

The 20 individual bay compatibility conditions, each of which involves six stresses, have the following cyclic form:

$$
\begin{gathered}
\tilde{c}_{2,1} \sigma_{1}+\tilde{c}_{2,2} \sigma_{2}+\tilde{c}_{2,3} \sigma_{3}+\tilde{c}_{2,4} \sigma_{4}+\tilde{c}_{2.5} \sigma_{5}+\tilde{c}_{2, \mathrm{~h}} \sigma_{6}=0 \\
\quad(10 \mathrm{~b} .1 \\
\tilde{c}_{3,6} \sigma_{6}+\tilde{c}_{3,7} \sigma_{7}+\tilde{c}_{3,8} \sigma_{8}+\tilde{c}_{3,9} \sigma_{9}+\tilde{c}_{3,10} \sigma_{10}+\tilde{c}_{3,11} \sigma_{11}=0 \\
\dot{\cdot} \\
\dot{\cdot}(10 \mathrm{~b} .2 \\
\tilde{c}_{21,96} \sigma_{96}+\tilde{c}_{21,97} \sigma_{97}+\tilde{c}_{21,98} \sigma_{98}+\tilde{c}_{21,99} \sigma_{99}+\tilde{c}_{21.100} \sigma_{100} \\
+\tilde{c}_{21.101} \sigma_{101}=0 \quad(10 \mathrm{~b} .20
\end{gathered}
$$


TABLE II--RANKS OF SEVERAL CONSTRAINT-GRADIENT MATRICES FOR

THE. 20-BAY TRUSS

|The element areas are pseudorandom numbers between 1.0 and 11.0 in. $\left.{ }^{2}\right]$

\begin{tabular}{|c|c|c|c|c|}
\hline \multirow[t]{2}{*}{ Case } & \multicolumn{2}{|r|}{ Constraint sest } & \multirow{2}{*}{$\begin{array}{l}\text { Rank of } \\
\text { constraint- } \\
\text { gradient } \\
\text { matrix }\end{array}$} & \multirow{2}{*}{$\begin{array}{l}\text { Nature of } \\
\text { singularity }\end{array}$} \\
\hline & Number & Constraints & & \\
\hline I & 181 & All stresses and displacements & 80 & Global \\
\hline 2 & 101 & AAll stresses? & 80 & Global \\
\hline 3 & 2 & $\left\{\sigma_{1}, X_{2} \mid\right.$ & 1 & Locial \\
\hline 4 & 3 & $\left|\sigma_{4}, X_{5}, X_{6}\right|$ & 2 & 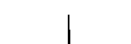 \\
\hline 5 & 5 & $a_{88}, X_{69}, X_{70}, X_{71}, X_{72}$ & 4 & \\
\hline 6 & 3 & $\left\{\sigma_{90}, X_{n y}, \sigma_{73 i}\right.$ & 2 & \\
\hline 7 & 6 & $\left|\sigma_{1}, \sigma_{2}, \ldots, \sigma_{6}\right|$ & 5 & \\
\hline 8 & 8 & $\sigma_{1}, \sigma_{2}, \ldots, \sigma_{6}, \sigma_{20}, X_{(x)}$ & 7 & \\
\hline 9 & 6 & $\sigma_{31}, \sigma_{32}, \ldots, \sigma_{36}$ & 5 & \\
\hline 10 & 20 & $\left.\sigma_{2}, \sigma_{7}, \sigma_{12}, \ldots, \sigma_{97}\right\}$ & 19 & 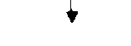 \\
\hline
\end{tabular}

Again, these relations (eq. (10)) are independent of the design variables. The sparsity, and (in the case of the 20 bay compatibility conditions) the bandedness, of the $[\tilde{C}]$ matrix is produced from the localized character of the relations. The external CC (eq. (10a)) ensures that the total deformation between the two boundaries (nodes 1 and 41 ) is zero (see fig. 2(b)). The 20 bay CC's ensure that the bars which form the bay deform in a consistent manner, such that all bars fit together before and after deformations, without inducing any residual strains (see, for example, fig. 2(c)).

Taking the first six stress constraints $\left\{\sigma_{1}, \sigma_{2}, \ldots, \sigma_{6}\right\}$ will produce a constraint-gradient matrix with a rank less than six (see case 7, table II). In fact, taking any set of constraints that include these six will produce a rank-deficient constraintgradient matrix (e.g., see case 8, table II). Other examples of singularities can be produced with sets of constraints that include $\left\{\sigma_{31}, \sigma_{32}, \ldots, \sigma_{36}\right\}$ or $\left\{\sigma_{2}, \sigma_{7}, \sigma_{12}, \ldots, \sigma_{97}\right\}$. These have also been verified numerically (see cases 9 and 10 , table II). Note, here, that the 20 stresses $\left\{\sigma_{2}, \sigma_{7}, \sigma_{12}, \ldots, \sigma_{97}\right\}$ correspond to the flexural load path for the structure under gravity loads and therefore are very likely to all become active, creating singularities during structural optimization procedures.

\section{Discussion}

We have seen how singularities can (sometimes quite easily) arise in structural optimization because of rank deficiencies in constraint-gradient matrices that are used in mathematical programming techniques to determine new search directions. In some variants of these algorithms, starting from an identity matrix, the matrix $[H]$ or related matrices are constructed by using constraint gradients following an update procedure (ref. 4). Often these matrices are initially well behaved, but become poorly conditioned (or singular) as the solution approaches the optimal point, thus requiring re-initialization. Noting that, typically, few constraints are active initially and that many may be active near the optimal solution, it is reasonable to hypothesize that the singularities observed are due to linear dependence among the active constraints. Once again, it seems very important to ensure that the active constraints chosen constitute a linearly independent set of functions.

Although generating conditions in which singularities can arise is very easy using equations (8), producing an algorithm to ensure linear functional independence among stress and displacement constraints is not so straightforward. One naive approach, which would be applicable to any structural analysis formulation, might be to find the rank of the matrix formed by the gradients of the active constraint set. If this matrix were rank deficient, the offending constraint(s) could be deleted, or replaced with some "less active, " but (what one would hope are) independent, constraint(s).

A far less costly, and more elegant, approach would be to examine the stress-displacement relations, the displacementstress relations, and the compatibility conditions of the IFM (eqs. (8)). Although the stress-displacement relations alone would be sufficient, it would often be advantageous to use one, or both, of the other two relationships. For example, when only stress constraints occur in the active constraint set. examination of the $\mathrm{CC}$ alone would be sufficient to determine whether or not the set was linearly functionally independent. Details on a procedure to ensure linear functional independence among active constraints will not be given here, but will be provided in a subsequent paper (Guptill, J.D.; Patnaik, S.N.: and Berke, L.: Identification and Preclusion of Singularities during Structural Optimization; to be published).

\section{Multiple Load Conditions}

With multiple load conditions that are mutually independent, it can be shown that the stress gradients for any single element are mutually independent. To illustrate this result with a simple numerical example, we took two load conditions for the three- 


\section{TABLE III.-RANKS OF SEVERAL CONSTRAINT GRADIENT MATRICES FOR MULTIPLE LOAD CONDITIONS}

|Element areas: 1.00. 9.30, and 3.67 in. ${ }^{2}$

\begin{tabular}{|c|c|c|c|c|}
\hline \multirow[t]{3}{*}{ Case } & \multicolumn{3}{|c|}{ Constraint stet } & \multirow{3}{*}{$\begin{array}{c}\text { Rank of } \\
\text { constraint- } \\
\text { gradicnt } \\
\text { matrix }\end{array}$} \\
\hline & \multirow[t]{2}{*}{ Number } & \multicolumn{2}{|c|}{$\begin{array}{c}\text { Load } \\
\text { conditions" }\end{array}$} & \\
\hline & & & 2 & \\
\hline 1 & 2 & $\left|\sigma_{1}\right|$ & $\left|\sigma_{1}\right|$ & 2 \\
\hline 2 & 2 & $\left|\sigma_{2}\right|$ & $X_{2}$ & 2 \\
\hline 3 & 3 & $\left|\sigma_{1}, \sigma_{2}\right|$ & $\left.\sigma_{3}\right\}$ & 3 \\
\hline
\end{tabular}

aload cenditens are definced in figure

bar truss shown in figure 1. The linear independence of the stress gradients associated with the first element under the two load conditions is demonstrated in table III. Furthermore, although $\sigma_{2}$ and $X_{2}$ were found to be linearly functionally dependent under one load condition, taking $\sigma_{2}$ from load condition 1 and $X_{2}$ from load condition 2 produces a gradient matrix of full rank (see table III). Moreover, taking two linearly functionally independent constraints from one load condition and one from another produces a gradient matrix of rank three. An example of this is also shown in table III.

\section{Frequency Constraints}

Frequency constraints appear to be linearly functionally independent of stress and displacement constraints. The frequency constraint $f$ is inversely proportional to the frequency $\omega$ where

$$
\omega^{2}=\frac{\vec{X}_{\omega}^{T}[K] \vec{X}_{\omega}}{\vec{X}_{\omega}^{T}[M] \vec{X}_{\omega}}=\frac{\vec{F}_{\omega}^{T}[S] \vec{F}_{\omega}}{\vec{F}_{\omega}^{T}\left[M^{\top}\right] \vec{F}_{\omega}}
$$

and $\vec{X}_{\omega},[K]$, and $[M]$ are the displacement mode shape, the stiffness matrix, and the mass matrix, respectively, associated with the displacement method (ref. 8); and $\vec{F}_{\omega},[S]$, and $\left[M^{7}\right]$ are the force mode shape, the IFM governing matrix, and the IFM mass matrix, respectively (ref. 9). It seems unlikely that the frequency constraint could ever be written as a linear combination of the stress and displacement constraints. Numerical examples indicating the linear independence of frequency constraint gradients are shown in Table IV.

\section{Structures Other Than Trusses}

For nontruss structures, the complexity is increased for several reasons, including (1) the presence of more than one design variable per element, (2) the presence of multi-axial
TABLE IV. -RANKS OF SEVERAL CONSTRAINT-GRADIENT MATRICES IN THE PRESENCE OF FREQUENCY CONSTRAINTS [Element areas: 1.00, 9.30. and 3.67 in. ${ }^{2}$ ]

\begin{tabular}{|c|c|c|c|c|}
\hline Case & \multicolumn{3}{|c|}{ Constraint set } & \multicolumn{2}{c|}{$\begin{array}{c}\text { Rank of constraint- } \\
\text { gradicnt matrix }\end{array}$} \\
\cline { 2 - 5 } & Number & Constraints & $\begin{array}{c}\text { With } \\
\text { frequency } \\
\text { constraints }\end{array}$ & $\begin{array}{c}\text { Without } \\
\text { frequency } \\
\text { constraints }\end{array}$ \\
\hline 1 & 6 & $\left\{\sigma_{1}, \sigma_{2}, \sigma_{3}, X_{1}, X_{2} f\right\}$ & 3 & 2 \\
$\left\{\sigma_{1}, \sigma_{2}, \sigma_{3} f\right\}$ & 3 & 2 \\
3 & 4 & $3 \sigma_{2}, X_{2} f$ & 2 & 1 \\
\hline
\end{tabular}

stress states, and (3) more complicated IFM equations. An initial introduction to the complexity of singularity issues for nontruss structures has been reported (ref. 9).

\section{Conclusions}

Singularity conditions can arise in structural optimization because of linear functional dependence among active stress and displacement constraints. These conditions can be global or local in nature. Local singularities can occur more frequently than global singularities. Linear functional dependence can be seen among sets of constraints containing very small percentages of the prescribed behavior constraints.

The presence of linear functional dependence can best be determined by examination of the equations derived in the integrated force method. If the active constraint set is composed of stress constraints only, examination of the IFM compatibility conditions is sufficient. When the active constraint set includes both stress and displacement constraints. either the stress-displacement relations or a combination of displacement-stress relations and the compatibility conditions need to be examined. Frequency constraints appear to be linearly functionally independent of the stress and displacement constraints. Stress and displacement constraints that cross multiple (mutually independent) load conditions are linearly functionally independent. Although more complicated, it would be of benefit to extend this analysis of singularities to nontruss structures.

Lewis Research Center

National Aeronautics and Space Administration Cleveland, Ohio, November 12, 1991 


\section{Appendix A \\ Symbols}

$A_{i} \quad$ cross-sectional area of the $i$ th element

$[B] \quad$ equilibrium matrix

$[\tilde{B}] \quad$ modified equilibrium matrix

$\tilde{b}_{i, j} \quad(i, j)$ th element of the modified equilibrium matrix

$[C]$ compatibility matrix

$[\tilde{C}] \quad$ modified compatibility matrix

$\tilde{c}_{i, j} \quad(i, j)$ th element of the modified compatibility matrix

E Young's modulus

$E_{i} \quad$ Young's modulus of the $i$ th element

$\left[E_{i}\right]$ diagonal matrix of Young's moduli scaled with lengths

$\vec{F} \quad$ internal forces

$\vec{F}_{\omega} \quad$ force mode shape associated with IFM frequency analysis

$f \quad$ frequency constraint

$[G]$ concatenated flexibility matrix

$g_{j} \quad j$ th constraint value

[H] coefficient matrix in a method of feasible directions

[I] identity matrix

$[J] \quad$ deformation coefficient matrix

$[\tilde{J}] \quad$ modified deformation coefficient matrix

$[K] \quad$ stiffness matrix associated with the displacement method

$\ell \quad$ characteristic dimension of the three-bar truss

$\ell_{i} \quad$ length of the $i$ th element

$[M]$ mass matrix associated with the displacement method

\section{$\left[M^{\top}\right] \quad$ IFM mass matrix}

$m \quad$ number of displacement degrees of freedom

$n \quad$ number of design variables; number of stress degrees of freedom

$\vec{P} \quad$ load vector
$\vec{P}^{*} \quad$ modified load vector

$r \quad$ number of strain compatibility conditions

$[S] \quad$ IFM governing matrix

$\vec{X} \quad$ displacement vector

$X_{j} \quad j$ th nodal displacement

$X_{\omega} \quad$ displacement mode shape associated with the displacement method

$\left\langle\alpha_{j}\right\} \quad$ set of constants

$\vec{\beta} \quad$ deformation vector

$\vec{\beta}_{0} \quad$ initial deformation vector

$\delta \vec{R} \quad$ effective initial deformation vector

$\iota \quad$ node number

$\kappa \quad$ number of active constraints used in the calculation of the direction vector

$\lambda_{0} \quad$ scaling factor

$\vec{\sigma} \quad$ stress vector

$\sigma_{0} \quad$ strength

$\sigma_{i} \quad$ stress of the $i$ th element

$\vec{x} \quad$ design variable vector

$\chi_{i} \quad i$ th design variable

$\vec{\varphi} \quad$ direction vector

$\omega \quad$ frequency

$\overrightarrow{0} \quad$ null vector

$\vec{\nabla} f \quad$ gradient of the merit function

$\lceil\nabla g] \quad$ constraint-gradient matrix

$\nabla g_{j} \quad$ gradient of the $j$ th constraint

$\partial g_{j} / \partial \chi_{i}$ partial derivative of the $j$ th constraint with respect to the $i$ th design variable

$[\cdot]^{T} \quad$ transpose of a matrix

$[\cdot]^{-1} \quad$ inverse of a matrix

$[\cdot]^{-r}$ inverse transpose of a matrix 


\section{Appendix B \\ Key Integrated Force Method Equations}

The key equations of the integrated force method (IFM) as related to the singularity issue are presented in this appendix. The IFM considers all the internal $n$ forces, $\vec{F}$, as the simultancous unknowns. The $m$ force equilibrium equations, $[B] \vec{F}=\vec{P}$, and the $r$ strain compatibility conditions, $[C][G] \vec{F}=\overrightarrow{0}$, are concatenated to obtain the governing equations of the method (refs. 10 and 11) as

$$
\left[\frac{[B]}{\mid C][G]}\right] \vec{F}=\left[\frac{\vec{P}}{\delta \vec{R}}\right] \text { or }[S] \vec{F}=\vec{P}^{*}
$$

where $[B]$ is the $m \times n$ equilibrium matrix, $[C]$ is the $r \times n$ compatibility matrix, $[G]$ is the $n \times n$ concatenated flexibility matrix that links deformations $\vec{\beta}$ to forces $\vec{F}$ as $(\vec{\beta}=[G] \vec{F})$, $\vec{P}$ is the $m$-component load vector, $\delta \vec{R}$ is the $r$-component effective initial deformation vector defined as $\delta \vec{R}=-[C] \vec{\beta}_{0}$, $\vec{\beta}_{0}$ is the $n$-component initial deformation vector, $[S]$ is the $n \times n$ governing matrix, and $m+r=n$. The matrices $[B]$, $[C],[G]$, and $[S]$ are banded and have full row ranks of $m$, $r, n$, and $n$, respectively. For simplicity, initial deformations are neglected here $\left(\vec{\beta}_{0}=\overrightarrow{0}\right)$.

The solution of equation (B1) yields the $n$ forces, $\vec{F}$. The $m$ displacements, $\vec{X}$, are obtained from the forces by

$$
\vec{X}=[J][G \mid \vec{F}
$$

where $[J]$ is the $m \times n$ deformation coefficient matrix defined as $\left([J]=m\right.$ rows of $\left.[S]^{-T}\right)$. For static analysis, the matrix $[J]$ is independent of element areas. For trusses, the flexibility matrix $[G]$ is a diagonal matrix, and its elements are

$$
g(i, i)=\frac{\ell_{i}}{A_{i} E_{i}} \quad \text { for } i=1,2, \ldots, n
$$

where $\mathcal{l}_{i}$ is the length of the $i$ th element, $A_{i}$ is the area of the $i$ th element, and $E_{i}$ is the Young's modulus of the $i$ th element.

\section{Stress-Displacement Relations}

The stress-displacement relations can be obtained from the general relations $\left(\vec{\beta}=[G] \vec{F}=[B]^{7} \vec{X}\right)$ with appropriate specialization for trusses as

$$
\vec{\sigma}=[\tilde{B}] \vec{X}
$$

where $[\tilde{B}]$ is a sparse, banded matrix with $[\tilde{B}]=\left[E_{\mathrm{l}} \| B\right]^{T}$, and $E_{i} / \ell_{i}$ are the nonzero elements of the diagonal matrix $\left[E_{i}\right]$.

\section{Displacement Stress Relations}

The displacements $\bar{X}$ can be written in terms of the stresses as

$$
\vec{X}=[\tilde{J}] \vec{\sigma}
$$

where $[\tilde{\eta}]=\left[J \mid E_{p}\right]^{-1}$.

\section{Compatibility Conditions in Terms of Stresses}

The strain compatibility conditions $([C][G] \vec{F}=\overrightarrow{0})$ can be written as stress compatibility conditions:

$$
[\tilde{C}] \vec{\sigma}=\overrightarrow{0}
$$

where $[\tilde{C}]$ is a sparse matrix with $[\tilde{C}]=[C]\left[E_{\ell}\right]^{-1}$. The $[\tilde{B}]$, $[\tilde{J}]$, and $[\tilde{C}]$ matrices can all be considered independent of element areas for static analysis. 


\section{References}

1. Patnaik, S.N.: Behavior of Trusses with Stress and Displacement Constraints. Comput. Struct., vol. 22, no. 4, 1986, pp. 619-623.

2. Patnaik, S.N.; and Dayaratnam, P.: Behavior and Design of PinConnected Structures. Int. J. Numer. Methods Eng., vol. 2, no. 4, 1970 , pp. $579-595$.

3. Dayaratnam, P.; and Patnaik. S.N.: Feasibility of Full Stress Design. AIAA J., vol. 7, no. 4, 1969, pp. 773-774.

4. Gallagher, R.H.; and Zienkiewicz, O.C., eds.: Optimum Structural Design. John Wiley \& Sons, 1973.

5. Patnaik, S.N.; and Gallagher, R.H.: Gradients of Behavior Constraints and Reanalysis Via the Integrated Force Method. Int. J. Numer. Methods Eng., vol. 23, no. 12, 1986, pp. 2205-2212.

6. Patnaik, S.N.: Berke. L.: and Gallagher, R.H.: Integrated Force Method Versus Displacement Method for Finite Element Analysis. Comput. Struct., vol. 38, no. 4, 1991, pp. 377-407.
7. Reklaitis, G.V.; Ravindran, A.; and Ragsdell, K.M.: Engineering Optimization: Methods and Applications. John Wiley \& Sons. 1983.

8. Zienkiewicz, O.C.: The Finite Element Method in Engineering Science. McGraw-Hill, New York. 1977.

9. Patnaik, S.N.; and Yadagiri, S.Y.: Design for Frequency by the Integrated Force Method. Comput. Methods Appl. Mech. Eng., vol. 16, no. 2 . 1978, pp. 213-230.

10. Patnaik, S.N.; Berke, L.; and Gallagher, R.H.: Compatibility Conditions of Structural Mechanics for Finite Element Analyis. AIAA J. vol. 29, no. 5, 1991, pp. 820-829.

11. Patnaik, S.N.: Variational Energy Formulation for the Integrated Force Method. AIAA J., vol. 24, no. 1. 1986. pp. 129-137. 


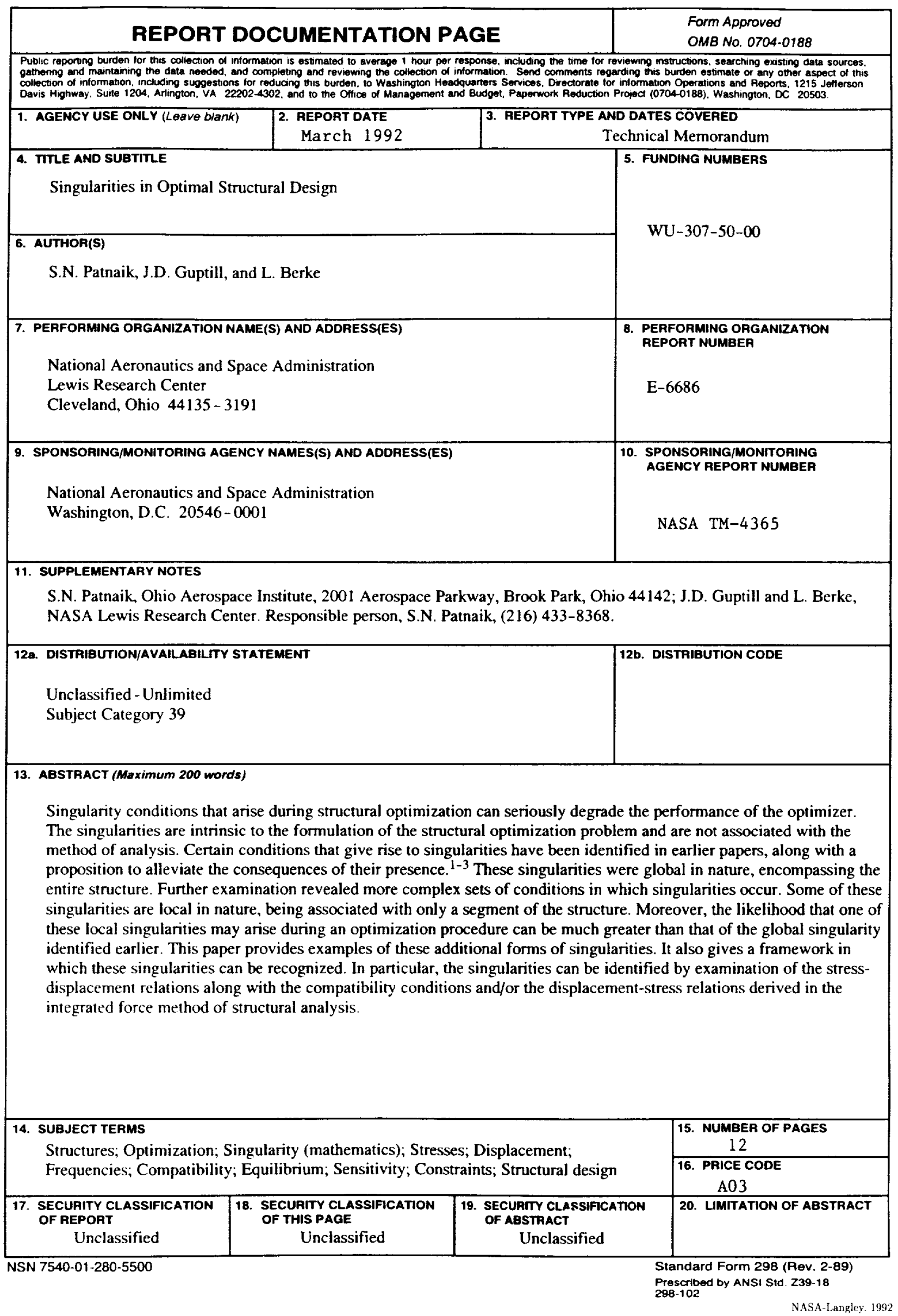

